\title{
Apport des neutrons à l'analyse structurale des composés partiellement désordonnés
}

\author{
A. Cousson
}

Laboratoire Léon Brillouin, CEA-CNRS, CE-Saclay, 91191 Gif-sur-Yvette cedex, France

\section{GENERALITES}

1.1 Spécificités des neutrons

1.2 Affinement

1.3 Synthèses de Fourier

1.4 Les macles

\section{EXEMPLES DE STRUCTURES PARTIELLEMENT DESORDONNEES}

2.1 Fullérène $\mathrm{C} 60$

2.2 Sulfure d'uranium $\alpha \mathrm{US}_{2}$

2.3 Désordre des protons des groupes méthyles

2.3.1 Le rotor quantique unidimensionnel

2.3.2 Les trihalogénomésitylènes

2.3.3 La 2,6-diméthylpyrazine

2.3.4 L'acétate de Lithium dihydraté

2.3.5 Le diacétate de Manganèse tétrahydraté

2.3.6 La g-picoline

\section{CONCLUSION}

\section{REFERENCES}

\section{RESUME}

La cristallographie est un outil extrêmement puissant qui pourrait être utilisé par de nombreux scientifiques dont les sujets de recherche sont en fait très éloignés. L'évolution des techniques ces dernières années a relégué par exemple la cristallographie des rayons $\mathrm{X}$ des petites molécules à un rôle mineur, un rôle de service. Certains ont même le sentiment semble-t-il que toutes les connaissances sont contenues dans de multiples logiciels capables par eux-mêmes de conduire une analyse structurale à un résultat correct unique. Il est souhaitable que chacun soit capable de réaliser l'étude structurale du composé qui l'intéresse et bien entendu nécessaire de comprendre ce que l'on fait, la qualité des résultats et leur analyse en dépend. L'objet de cette présentation est de montrer l'apport spécifique de la diffraction de neutrons sur monocristaux à l'étude du désordre, en particulier des atomes d'hydrogène, et ses conséquences sur la compréhension des propriétés physiques, à partir de développements et d'exemples récents. 


\section{GENERALITES}

Une étude structurale se déroule en plusieurs étapes : la première étape, pas toujours triviale, consiste à obtenir un monocristal, la seconde consiste à réaliser une expérience de diffraction, le but est de mesurer le maximum possible raisonnable d'intensités diffractées. Cette seconde étape permet de déterminer la métrique du système, la symétrie et le groupe d'espace. L'étape suivante est la détermination et l'affinement de la structure, et la dernière étape consiste à interpréter et analyser les résultats obtenus.

La détermination de la structure est pratiquement dans tous les cas réalisée à partir des données $\mathrm{X}$. Cette détermination est également possible à partir de données neutrons, souvent pour le plaisir.

\subsection{Spécificité des neutrons}

La différence entre rayons $X$ et neutrons dépend de plusieurs facteurs : taille de l'échantillon, facteur de diffusion, absorption entre autres. Les rayons $X$ interagissent avec les électrons, les neutrons avec les noyaux. Les longueurs de diffusion des noyaux sont comprises entre les valeurs extrêmes de $-3.74 \mathrm{fm}$ pour l'hydrogène et +12.29 pour le scandium. Vu par les neutrons l'azote ( 7 électrons, $b=9.36 \mathrm{fm}$ ) est plus lourd que l'uranium qui avec ses 92 électrons a une longueur de diffusion de $8.417 \mathrm{fm}$. L'absorption est en général plus importante avec des rayons $\mathrm{X}$, c'est vraiment le cas de l'uranium mais par exemple le plutonium absorbe peu à la longueur du molybdène. Il est donc plus facile de réaliser une expérience rayons $\mathrm{X}$ sur un composé de plutonium que d'uranium, quoique. L'absorption dans le cas des neutrons peut être importante, c'est la cas de B, Cd et de certaines terres rares, il reste alors la possibilité d'utiliser des isotopes non absorbants. Cette particularité propre aux neutrons est très souvent exploitée lorsqu'on étudie par exemple des composés fortement hydrogénés où la diffusion incohérente de l'hydrogène pourrait poser problème, on remplace alors l'hydrogène par le deutérium de spin entier, $b_{H}=-3.74 \mathrm{fm}$ et $b_{D}=6.671 \mathrm{fm}$. L'autre aspect très important est qu'une densité électronique est toujours positive, ce qui n'est pas le cas d'une densité nucléaire. Enfin, le noyau est un objet ponctuel et ne présente donc pas de facteur de forme en fonction de l'angle de diffraction.

L'avantage des neutrons est clair si on s'intéresse au désordre des atomes légers et en particulier au désordre des protons. Bien qu'évident puisqu'on aura à étudier surtout la partie négative de la densité, où on ne pourra trouver que les protons, cet avantage sera un peu atténué par la faible valeur de la longueur de diffusion du proton, rendue encore plus faible par sa délocalisation éventuelle, cette faible valeur redevient un avantage si on considère que généralement le poids des protons modifie rarement la phase du facteur de structure.

\subsection{Affinement}

Le but d'une étude structurale est de fournir le plus précisément possible les paramètres des positions atomiques $(\mathrm{x}, \mathrm{y}, \mathrm{z})$ et leurs paramètres d'agitation thermique, isotrope ou anisotrope. Le résultat dépend du nombre et de la qualité des données observées. En ne considérant que le nombre de données observées on souhaite qu'il y ait au moins dix facteurs de structure observés par paramètre variable.

Dans le cas général le facteur de structure pour un ensemble d'atomes discrets est proportionnel à l'expression suivante :

$$
F_{h k l}=\Sigma \exp \left(-B_{j} \sin ^{2} \theta / \lambda^{2}\right) f_{j} \exp \left\{2 \pi i\left(h x_{j}+k y_{j}+l z_{j}\right)\right\}
$$


La somme est étendue à tous les atomes $\mathrm{j}$ de la maille occupant les positions $\mathrm{x}_{\mathrm{j}}, \mathrm{y}_{\mathrm{j}}, \mathrm{z}_{\mathrm{j}}$, de facteur de diffusion $f_{j}$ et de paramètre thermique $B_{j}$.

Or il est fréquent qu'un atome n'occupe pas une position bien définie dans la maille élémentaire, il peut être réparti sur plusieurs positions, discrètes ou continues. Si le nombre de positions est finie il est possible d'affiner ces positions avec des taux d'occupation partiels. Décrire des distributions continues à partir de positions discrètes conduit très vite à un très grand nombre de paramètres et comme souvent dans les systèmes désordonnés le nombre d'observations est réduit ou de qualité moyenne l'affinement du modèle devient très difficile. Quelquefois il est possible de paramétrer le désordre avec un petit nombre de paramètres et entrer ces paramètres comme variables dans l'affinement. Pour cela il est préférable de décrire le désordre par une figure géométrique correspondant à l'espace occupé par les atomes. Ce travail a déjà été réalisé précédemment pour trois cas particuliers de figures et nous l'avons généralisé à toutes les situations possibles, quelles que soient l'orientation des figures géométriques et le groupe d'espace. L'expression du facteur de structure d'une figure spéciale s'ajoute au facteur de structure écrit précédemment. Pour un ensemble d'atomes distribués sur une sphère le facteur de structure s'écrit :

$$
\mathrm{S}(\mathrm{H}, \mathrm{x})=\mathrm{S}(\mathrm{H}, \mathrm{R})=\sin 2 \pi \mathrm{HR} / 2 \pi \mathrm{HR}
$$

avec $H=2 \sin \theta / \lambda$ et $\mathrm{R}$ rayon de la sphère.

Dans le cas d'atomes répartis uniformément le long d'une ligne :

$$
\mathrm{S}(\mathrm{H}, \mathrm{x})=\sin \mathrm{p} / \mathrm{p}
$$

avec $\mathrm{p}=2 \pi \mathrm{Ha} \cos \psi$

et dans ce $\operatorname{cas} \mathrm{H}=2 \sin \theta / \lambda$,

2 a représente la longueur de la ligne,

$\psi$ est l'angle entre la ligne et $\mathrm{H}$ (pour chaque réflexion).

Dans le cas d'une distribution annulaire l'expression du facteur de structure s'écrit :

avec $\mathbf{J}_{0}$ : fonction de Bessel d'ordre 0,

$$
\mathrm{S}(\mathrm{H}, \mathrm{x})=\mathrm{J}_{0} 2 \pi \mathrm{HR} \sin \psi
$$

$\mathrm{H}=2 \sin \theta / \lambda$,

$\mathrm{R}$ rayon de l'anneau

et $\psi$ angle entre le vecteur normal au plan de l'anneau et $\mathrm{H}$ (c'est à dire avec chaque réflexion).

Ces possibilités ont été introduites dans CRYSTALS, testées et rendues simples à utiliser. En général dans un affinement par moindres carrés plus on introduit de paramètres plus l'accord entre calcul et observation s'améliore. Des tests statistiques permettent de rendre compte de l'amélioration effective de cet accord. Dans tous les cas que nous avons étudiés les tests se sont avérés inutiles puisqu'avec un nombre réduit de paramètres on a toujours obtenu un meilleur accord entre facteurs de structures observés et calculés. Par exemple pour décrire, dans le cas général, une distribution annulaire on affine cinq paramètres, tandis qu'à partir de positions discrètes on introduit au minimum six atomes anisotropes donc 54 paramètres et néanmoins le résultat est meilleur dans le premier cas. Cet accord est calculé sur l'ensemble des réflexions observées : $\mathrm{R}=\mathrm{S}|| \mathrm{F}_{\mathrm{o}}|-| \mathrm{F}_{\mathrm{c}}|/ \Sigma| \mathrm{F}_{\mathrm{o}} \mid$. 


\subsection{Synthèses de Fourier}

L'expression générale du calcul de la densité dans la maille s'écrit :

$$
\rho(\mathrm{r})=1 / \mathrm{V} \Sigma \mathrm{F}_{\mathrm{h}} \exp \{-2 \pi(\mathrm{h} . \mathrm{r})\}
$$

ou

$$
\rho(\mathrm{x}, \mathrm{y}, \mathrm{z})=1 / \mathrm{V} \Sigma \Sigma \Sigma \mathrm{F}_{\mathrm{hk}} \exp \{-2 \pi \mathrm{i}(\mathrm{hx}+\mathrm{ky}+\mathrm{lz})\}
$$

Connaissant les facteurs de structure on est donc capable de calculer la densité électronique ou nucléaire, positive ou négative, en tout point de la maille. Dans le cas de systèmes désordonnés ou pour une structure partiellement résolue un excellent moyen de mise en évidence du désordre ou de résidus éventuels consiste à calculer une série différence à partir de l'expression :

$$
\Delta \rho=1 / \mathrm{V} \Sigma \Sigma \Sigma\left(\left|\mathrm{F}_{\mathrm{o}}\right|-\left|\mathrm{F}_{\mathrm{c}}\right|\right) \exp \left(\mathrm{i} \alpha_{\mathrm{c}}\right) \exp \{-2 \pi \mathrm{i}(\mathrm{hx}+\mathrm{ky}+\mathrm{lz})\}
$$

où $\alpha_{c}$ est la phase de $F_{c}$. La série différence a deux avantages principaux. D'une part, si les phases $\alpha_{\mathrm{c}}$ calculées sont égales aux phases observées $\alpha_{0}$ elle fournit une mesure directe des erreurs entre le modèle utilisé et la vraie structure contenue dans les valeurs observées $\left|F_{0}\right|$. Cette propriété est très utile dans les premières étapes d'affinement d'un modèle. D'autre part, plus important encore, même si les phases sont modérément fausses la Fourier $\Delta \mathrm{F}$ peut fournir une information utile qui est difficile ou impossible à obtenir à partir d'une transformée de Fourier sur les $\mathrm{F}_{0}$. Il est nécessaire de signaler qu'à la fin d'une étude on calcule les résidus en électrons $/ \AA^{3}$ ou en $\mathrm{fm} / \AA^{3}$ à l'échelle absolue, ultime vérification du modèle affiné, pas seulement pour voir si on a rien oublié. Ce calcul dépend du facteur d'échelle et de la valeur de la réflexion $\mathrm{F}_{000}$ qui n'est pas mesurable. On peut cependant montrer qu'elle est approximativement égale à la somme des électrons contenus dans la maille dans le cas des rayons $\mathrm{X}$ et à la somme des longueurs de diffusion dans le cas des neutrons.

\subsection{Les macles}

Par définition, deux cristaux sont maclés quand ils sont associés suivant une loi géométrique bien définie, et lorsque cette association géométrique se retrouve dans des cas fréquents. C'est le cas de nombreux minéraux : pyrite, quartz, fluorine, calcite par exemple. Les macles peuvent avoir été formées de plusieurs manières différentes. Deux types de macle nous concernent plus particulièrement :

1- les macles de transformation liées à une transition de phase, généralement du second ordre, qui est intervenue entre le moment où le cristal s'est formé et celui où on l'observe.

2- les macles de croissance. Il arrive qu'au cours de la croissance deux cristaux géométriquement associés croissent ensemble.

Ces macles sont malheureusement très fréquentes. Par diffraction de neutrons il est quelquefois possible de séparer les deux individus d'une macle de type 2, ce fut le cas par exemple pour un cristal de $\mathrm{C}_{60}$ et pour un cristal de $\mathrm{TlD}_{2} \mathrm{PO}_{4}$ à température ambiante. Dans le cas des macles de type 1 il faut faire avec, c'est le cas général des cuprates, supraconducteurs ou non, à température ambiante. Les composés moléculaires ne sont en général pas maclés à température ambiante. Mais pour ces composés il est intéressant de connaître très précisément la structure à basse température, typiquement en-dessous de $20 \mathrm{~K}$, domaine de température où la structure n'évolue plus et où on observe l'effet tunnel du proton. Entre la température ambiante et $20 \mathrm{~K}$ il n'est pas rare d'observer une ou deux transitions de phases. L'étude structurale est rendue plus difficile. A condition de pouvoir mesurer les intensités diffractées il 
faut également comprendre le maclage pour définir la loi de macle et à partir de la structure haute température établir un modèle structural. L'affinement du modèle, s'il est bien défini, n'est pas très différent d'un affinement classique si la macle est bien comprise. Le dernier point consiste, à partir du modèle affiné, à « démacler » le cristal si on souhaite observer par exemple comment est distribuée la densité par un calcul de Fourier, les intensités diffractées s'additionnent, pas les facteurs de structure. Dans les cas désespérés, désespérants où il est quasiment impossible de mesurer quoi que ce soit, il reste toujours la possibilité de faire appel à la diffraction sur poudres.

\section{EXEMPLES DES STRUCTURES PARTIELLEMENT DESORDONNEES}

Ce qui précède est maintenant illustré par quelques exemples d'atomes désordonnés à la surface d'une sphère $\left(\mathrm{C}_{60}\right)$, le long d'une ligne ( $\left.\alpha \mathrm{US} 2\right)$ ou en anneau (protons des $\mathrm{CH}_{3}$ ).

\subsection{Exemple d'atomes désordonnés à la surface d'une sphère : $\mathbf{C}_{60}$}

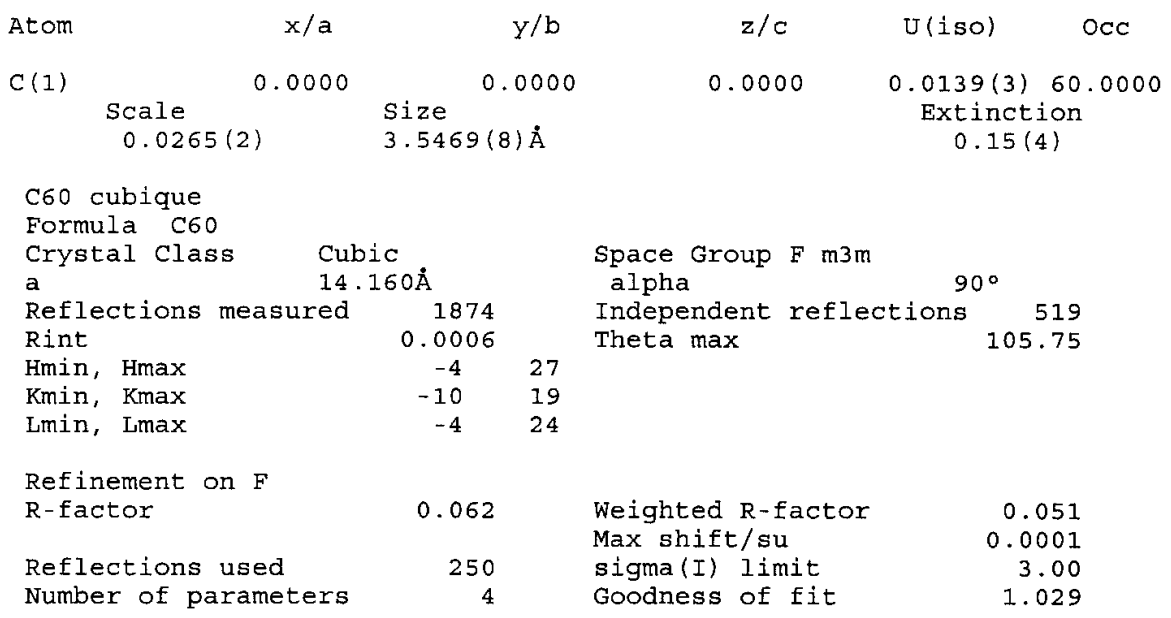

Le tableau ci-dessus résume les principales caractéristiques de l'affinement du $\mathrm{C}_{60}$ à partir de données de diffraction de neutrons sur un monocristal de $181 \mathrm{mg}$ (à peu près la moitié d'un monocristal maclé de $301 \mathrm{mg}$ ), la mesure a été effectuée jusqu'à $\sin \theta / \lambda=0.9604 \AA^{-1}$. Dans ce modèle les atomes de carbones sont répartis statistiquement à la surface d'une sphère de rayon $3.5469(8) \AA ̊$, l'extinction est significative mais faible, l'épaisseur de la sphère est donnée par le paramètre thermique affiné : $u[$ iso $]=0.0139(3) \AA^{2}$. Il est exclu dans ce cas d'imaginer un affinement à partir d'atomes de carbone occupant des positions discrètes, le résultat est remarquable si on considère qu'il n'a fallu que quatre paramètres.

Remarque : cet affinement n'apporte aucune information sur la dynamique des sphères ou sur une éventuelle anisotropie. 


\subsection{Exemple d'atomes désordonnés le long d'une ligne : $\alpha \mathbf{U S}_{2}$}

La structure de $\alpha \mathrm{US}_{2}$ a été déterminée par diffraction des rayons X. Le résultat de cette étude a révélé la présence d'une part d'une non-stoechiométrie due à des lacunes d'uranium et d'autre part d'un désordre sur le site de l'uranium concerné. Uranium et soufre sont deux éléments absorbants aux rayons $\mathrm{X}$, ceci a conduit quelques collègues à mettre en doute les résultats obtenus. Une expérience de diffraction de neutrons sur monocristal a alors été réalisée.

Le tableau ci-dessous correspond à un affinement classique qui montre bien une occupation partielle du site de l'atome d'uranium U(2) et une très forte anisotropie.

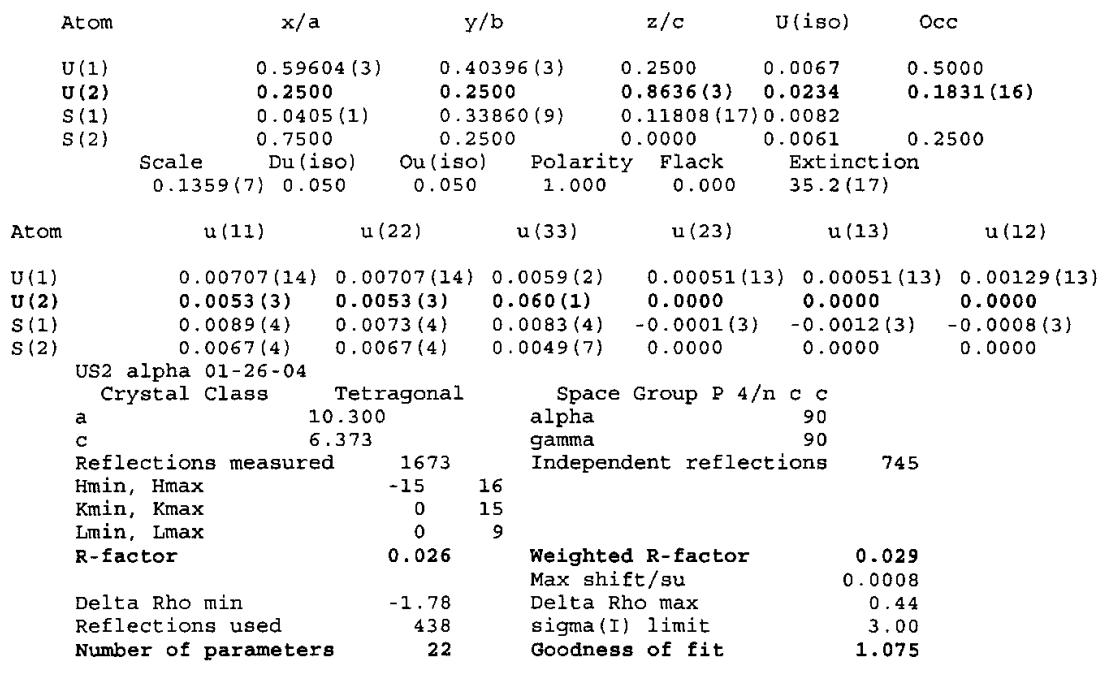

Un second affinement affinement a été réalisé en séparant en trois parties équivalentes l'atome d'uranium lacunaire, le seul résultat notable se traduit par une diminution de l'anisotropie.

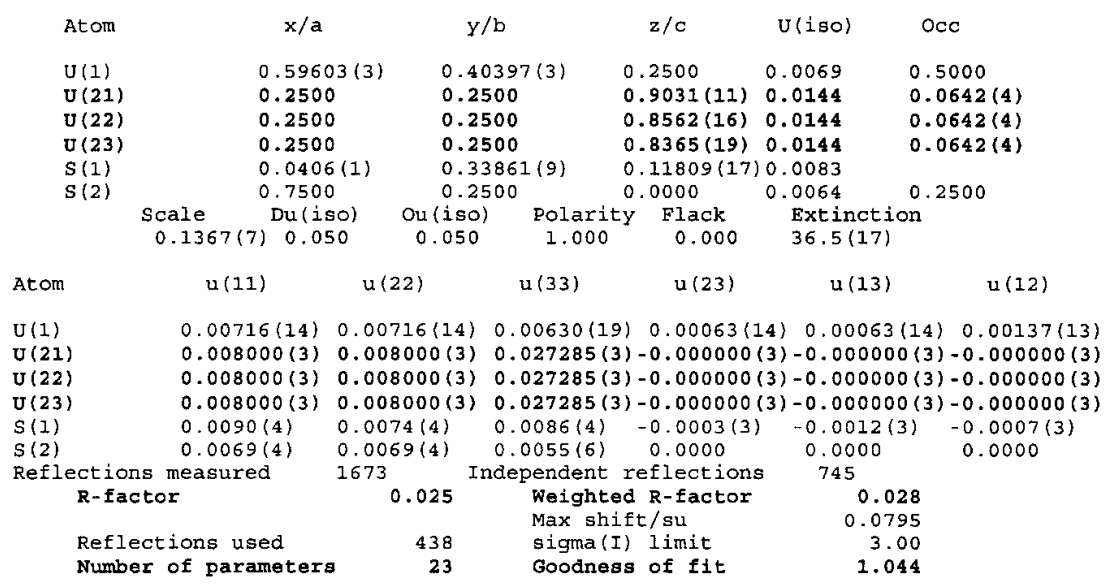


A partir des mêmes données et dans les mêmes conditions la structure a été affinée en considérant que l'atome d'uranium était réparti de manière continue sur un segment de droite. Cet affinement donne directement la longueur de la ligne (Size) occupée par l'uranium U(2) : $0.746(4) \AA$ et un paramètre thermique $u[$ iso $]=0.0056(3)$ comparable à celui de l'uranium non lacunaire.

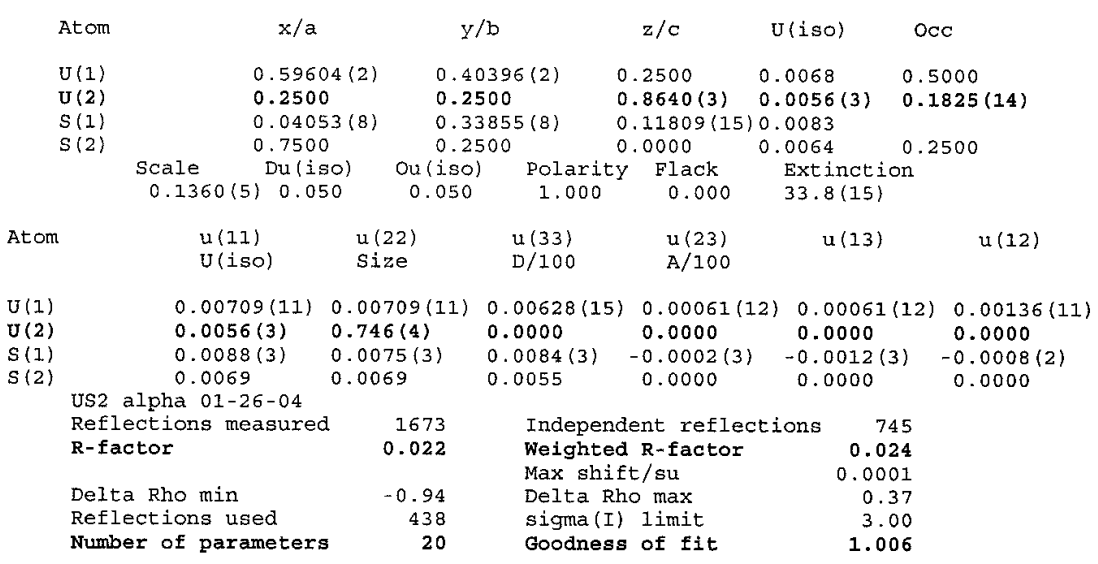

Bien que du point de vue du calcul proprement dit le résultat ne soit pas spectaculaire c'est tout de même dans ce dernier cas que le résultat est le meilleur. Cette expérience et cette description confirment de manière éclatante les résultats obtenus précédemment par diffraction des rayons $\mathrm{X}$.

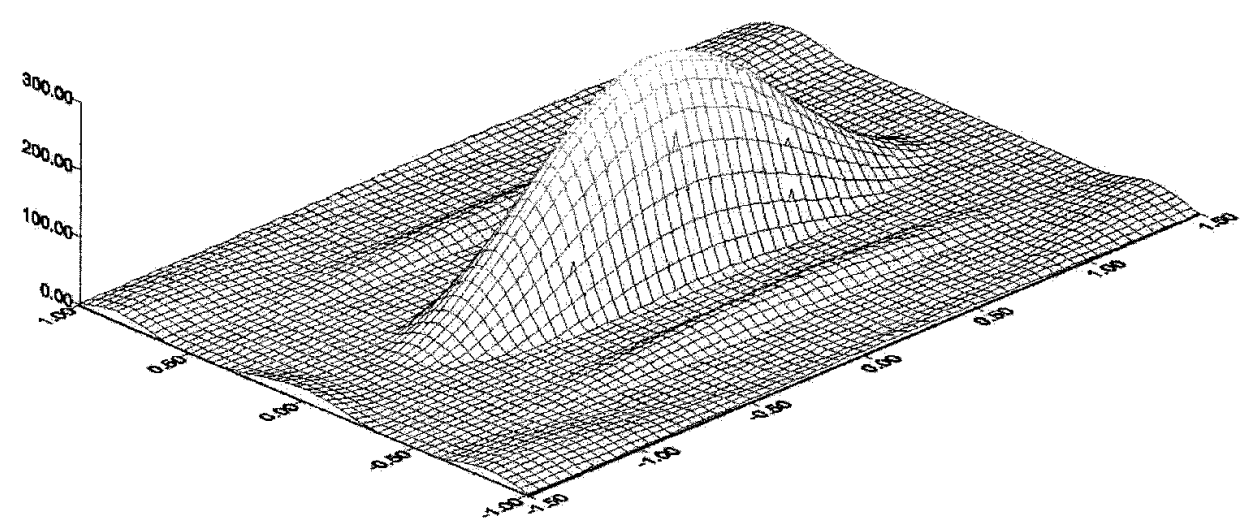

Distribution de la densité nucléaire de U(2) dans US2 alpha

Une série-différence de Fourier permet de visualiser la distribution de l'uranium. 


\subsection{Désordre des protons des groupements méthyles : effet tunnel des protons des $\mathrm{CH}_{3}$ dans les cristaux moléculaires.}

La diffraction de neutrons sur monocristaux, outre la structure, fournit une information importante sur la distribution des protons à toute température. Cependant, les contributions statistiques et dynamiques ne peuvent être a priori distinguées. Si on peut écarter une distribution statique des protons (due à une orientation aléatoire du méthyle), un modèle de distribution annulaire peut être interprété en terme de dynamique d'un rotateur libre (c'est à dire d'un rotateur faiblement gêné, barrière faible).

Une fois ce travail expérimental achevé, mise en évidence de la distribution des protons, il reste à comprendre les relations entre structure et dynamique. Les développements récents des méthodes de calcul quantique (calculs $a b$ initio) ou des potentiels empiriques, ainsi que les capacités de calcul des stations de travail permettent de calculer avec une précision sans cesse améliorée les potentiels locaux au sein d'un cristal. La comparaison des calculs et des données expérimentales permet de mieux comprendre l'origine des potentiels locaux en terme de grandeurs physico-chimiques.

La dynamique rotationnelle d'un $\mathrm{CH}_{3}$ faiblement gêné donne lieu à des transitions d'effet tunnel observables dans de nombreux cristaux moléculaires à très basse température $(\sim 2 \mathrm{~K})$. Les transitions observées dépendent seulement de la barrière de potentiel entre les configurations indiscernables obtenues par rotation de $\pm 2 \pi / 3$. Les transitions supérieures à $\sim 1$ $\mu \mathrm{eV}$ sont observables par diffusion inélastique des neutrons.

Dans le cristal, le potentiel provient de contributions intramoléculaires et intermoléculaires qui peuvent être distinguées. Le $1^{\text {er }}$ terme est déterminé par la géométrie du composé portant le $\mathrm{CH}_{3}$. La contribution intermoléculaire dépend de l'environnement du $\mathrm{CH}_{3}$ dans le cristal. En principe, ces contributions peuvent être reliées à la structure cristalline via des calculs de modélisation ( $a b$ initio ou empiriques). Et la grande sensibilité des mesures d'effet tunnel nous fournit un test de choix de la précision des différentes méthodes de calcul.

De plus, les transitions d'effet tunnel sont observées uniquement à basse température, elles disparaissent habituellement au dessus de $100 \mathrm{~K}$. Par conséquent la dynamique des méthyles et les fonctions potentielles à haute température sont inconnues. La diffraction de neutrons sur monocristaux est une technique unique pour fournir une densité de probabilité des protons à toute température. En principe, les calculs de modélisation sont nécessaires pour distinguer la contribution statique et la contribution dynamique. Pour le $\mathrm{CH}_{3}$ la dynamique domine largement la distribution des protons, ceci peut être confirmé en comparant la densité expérimentale et la distribution connue à basse température par les mesures d'effet tunnel. 


\subsubsection{Le rotor quantique unidimensionnel}

Le potentiel rotationnel s'écrit :

$V(\phi)=\sum_{n=1}^{N} \frac{V_{3 n}}{2}(1-\cos (3 n \phi))$

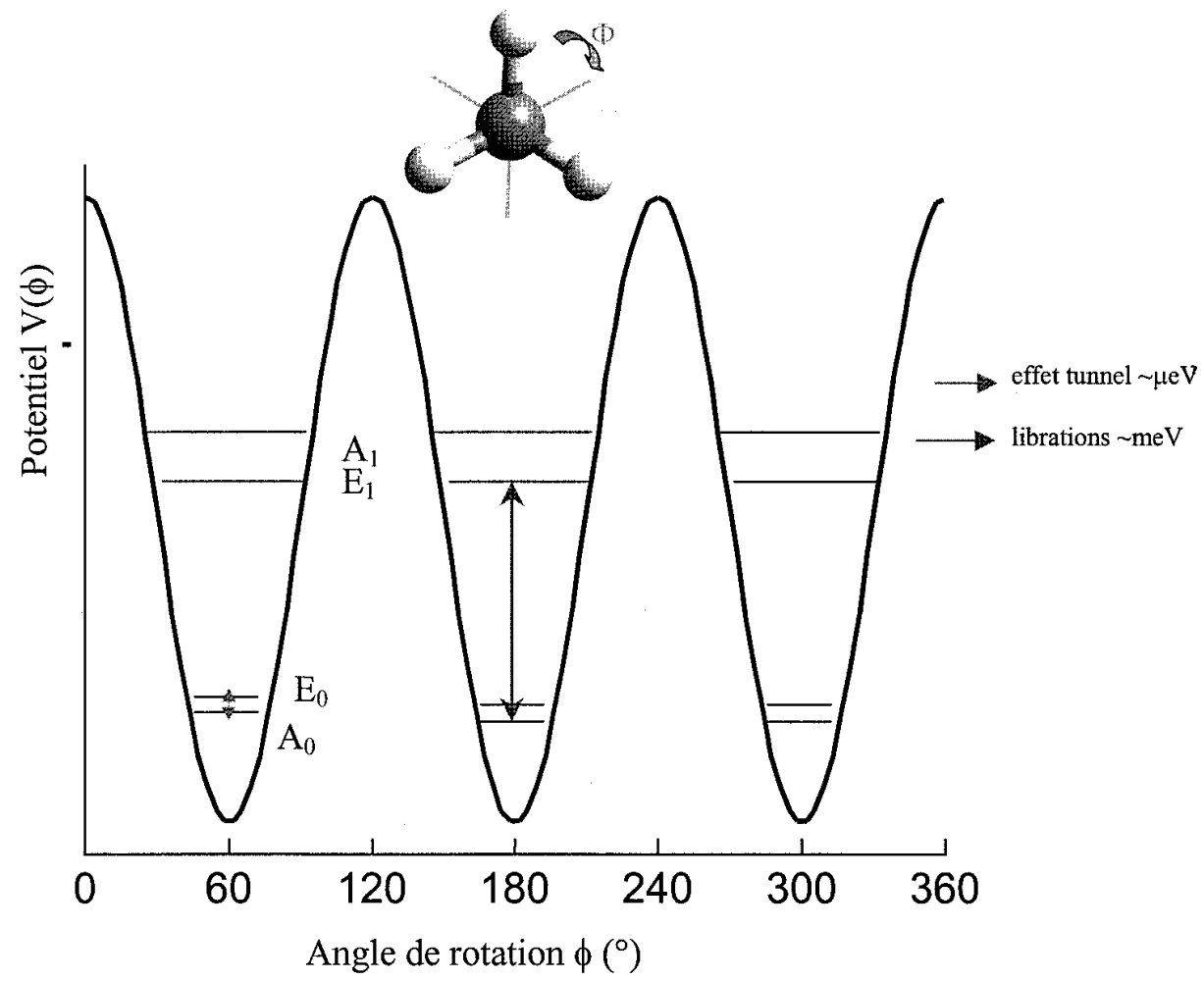

Exemple de potentiel $V_{3}$ et des niveaux d'énergie associés. L'échelle relative des énergies $n$ 'est pas respectée par souci de clarté. Les transitions observables par diffusion inélastique des neutrons sont représentées : $\omega_{01}$ représente une transition de libration et $\omega_{00}$ représente la transition d'effet tunnel dans l'état librationnel fondamental.

Dans le modèle de la particule isolée, le groupement méthyle est considéré comme un rotateur symétrique et rigide, dont l'environnement et l'axe de rotation sont fixes. L'énergie potentielle $\mathrm{V}(\phi)$ est fonction d'un seul paramètre : l'angle de rotation $\phi$. L'hamiltonien d'un tel système est représenté par :

$$
\boldsymbol{H}=-\boldsymbol{B} \frac{\partial^{2}}{\partial \phi^{2}}+V(\phi)
$$


$B$ est la constante de rotation et $I$ le moment d'inertie réduit, $B=0,655 \mathrm{meV}$ pour un groupement méthyle. La symétrie du groupement $\mathrm{CH}_{3}$ impose sa symétrie ternaire au potentiel. Les niveaux d'énergie et fonctions d'onde d'un rotateur gêné peuvent être calculés numériquement par diagonalisation de la matrice hamiltonien dans la base des fonctions d'onde d'un rotateur libre. Chaque niveau d'énergie, appelé niveau d'énergie librationnelle ou torsionnelle, est divisé en deux sous niveaux A et E. Deux types d'excitation sont mesurables: les transitions de libration, d'énergies comprises entre $\sim 2$ et $300 \mathrm{meV}$ et les transitions d'effet tunnel. Ce modèle relativement simple s'applique à un grand nombre de cas. Cependant des couplages ont été introduits pour tenir compte de la multiplicité des bandes parfois observées pour un seul groupement méthyle indépendant dans la maille cristalline, ou du déplacement des fréquences avec la température ou de la dilution isotopique. La suite de cet exposé concerne quelques cas qui illustrent l'apport de la diffraction de neutrons à l'étude de la dynamique des groupements $\mathrm{CH}_{3}$.

\subsubsection{Les trihalogéno-triméthyle-benzènes ou trihalogénomésitylènes}

La formule générale de ces composés est 1,3,5-trihalogéno-2,4,6-triméthyle-benzènes. Trois systèmes ont été étudiés: trichloro-(TCM), tribromo-(TBM) et tri-iodo-(TIM)triméthyle-benzène par diffusion inélastique des neutrons. Tous cristallisent dans le même groupe d'espace triclinique $\mathrm{P}-1$. Bien que la molécule libre apparaisse très symétrique la structure cristalline ne l'est pas du tout.

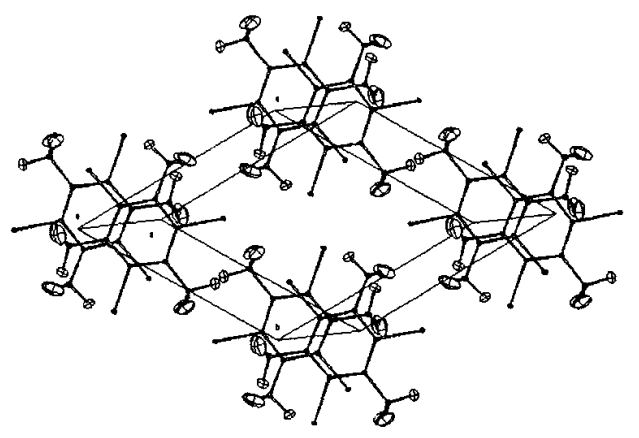

Structure du tribromo-triméthylebenzène à $14 \mathrm{~K}$.

Difficiles à étudier ces composés sont particulièrement intéressants et on se limitera ici aux tribromomésytilènes hydrogénés et deutérés. En ce qui concerne la diffraction de neutrons la principale difficulté est liée à la durée de l'expérience. Pour obtenir des données complètes, 
du fait de l'absence de symétrie, il est nécessaire de mesurer une demi-sphère de l'espace réciproque, ce qui représente, pour une expérience à une température donnée, un temps de mesure d'environ 15 à 20 jours. Ces composés ne présentent pas de transition de phase entre la température ambiante et les très basses températures. Les deux molécules de la maille sont reliées par un centre d'inversion, toutes les molécules sont empilées le long de l'axe a et sont parallèles entre elles. Quelques faits méritent d'être soulignés : la diffusion inélastique des neutrons fait apparaître trois fréquences tunnel différentes correspondant aux trois groupements méthyles indépendants; une expérience de diffusion inélastique des neutrons réalisée sur un gros monocristal orienté a permis d'attribuer à chaque groupement méthyle la fréquence qui lui correspond. C'est un des cas étudiés où les différents calculs de simulation rendent le mieux compte des expériences de diffusion inélastique en accord avec les résultats de l'étude structurale.

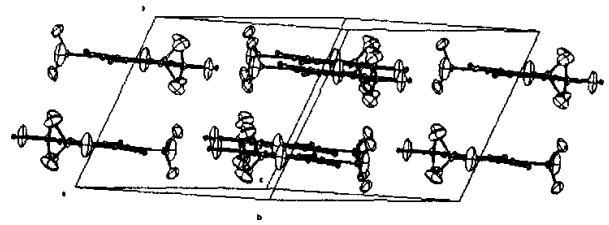

Représentation de la structure du tribromomésitylène hydrogéné à $14 K$, l'orientation correspond à celle du monocristal orienté pour l'expérience de diffusion inélastique des neutrons.

L'étude structurale à température ambiante montre une délocalisation de la distribution des protons des différents groupements $\mathrm{CH}_{3}$. En particulier les cartes de Fourier montrent une délocalisation avec quatre maxima plutôt que trois prévus. Dans le cas de ces composés, du fait de la présence du noyau benzénique, il est possible de traiter la molécule comme un corps rigide. Les paramètres thermiques anisotropes des atomes lourds deviennent les observables du modèle rigide. Il est alors possible de calculer les éventuels mouvements de rotation et de translation de ce corps rigide. En incluant le squelette carboné et les atomes de brome dans l'analyse on peut montrer que les librations sont importantes à température ambiante le long d'axes proches des axes moléculaires d'inertie et que ces librations sont pratiquement nulles à $14 \mathrm{~K}$. Cette absence de mouvement du corps rigide est certainement lié aux atomes lourds et explique également le bon accord entre simulation numérique et observation de l'effet tunnel dans ces systèmes puisque le potentiel vu par les protons n'est pas perturbé par l'agitation thermique du squelette.

Articles et thèses récentes de référence: $J$. Meinnel et al., thèses de F. Boudjada et $M$. Plazanet. 


\section{Tribromomésytilène deutéré}
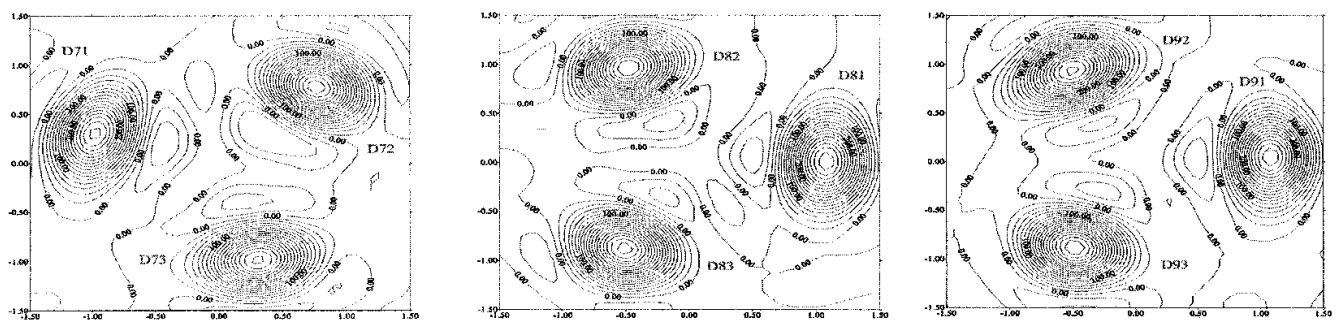

Ci-dessus : cartes de Fourier des trois $\mathrm{CD}_{3}$ indépendants à $14 \mathrm{~K}$.
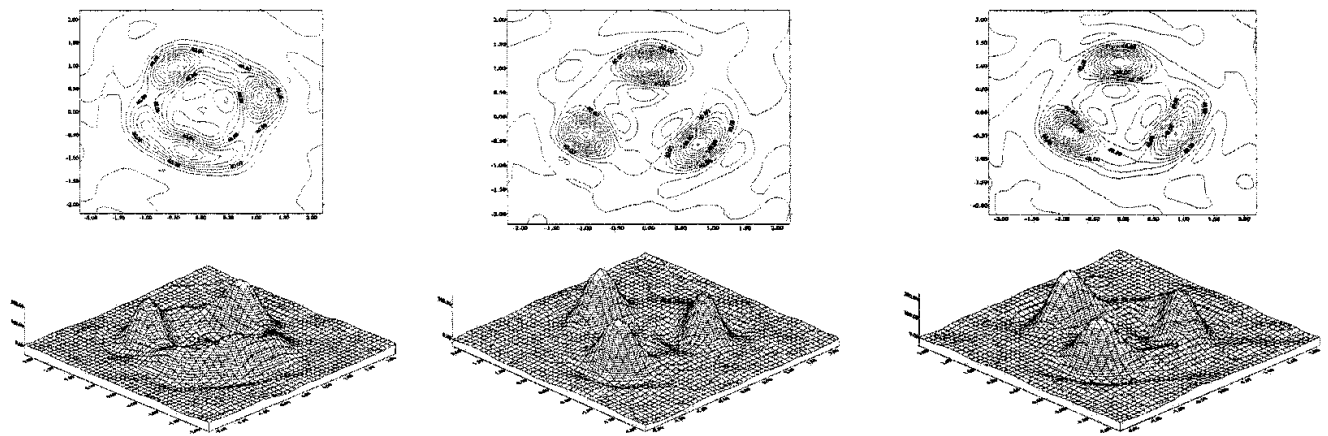

Représentation de la densité nucléaire des mêmes méthyles deutérés à $60 K$.
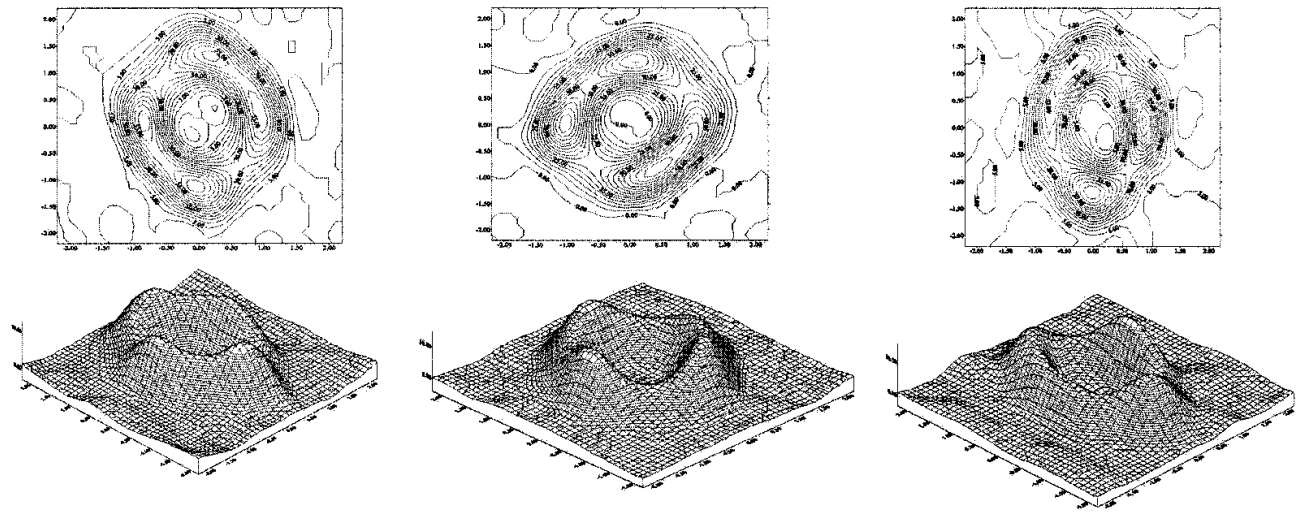

Cartes de Fourier à $295 \mathrm{~K}$ : dans le plan de l'anneau.

On voit clairement sur ces figures l'influence de la température sur la rotation des deutérons. A $14 \mathrm{~K}$ les sauts se font par effet tunnel, à $295 \mathrm{~K}$ la rotation est quasi libre. Les groupements deutérés $\mathrm{CD}_{3}$ sont également des rotateurs quantiques, leur moment d'inertie est simplement deux fois plus important que celui des $\mathrm{CH}_{3}$ et il peut être très intéressant de les comparer. 


\subsubsection{La 2,6-diméthylpyrazine}

indépendants.

Cette molécule possède deux groupements méthyle cristallographiquement

Les figures ci-dessous représentent cette molécule avec deux orientations différentes à $5 K$.
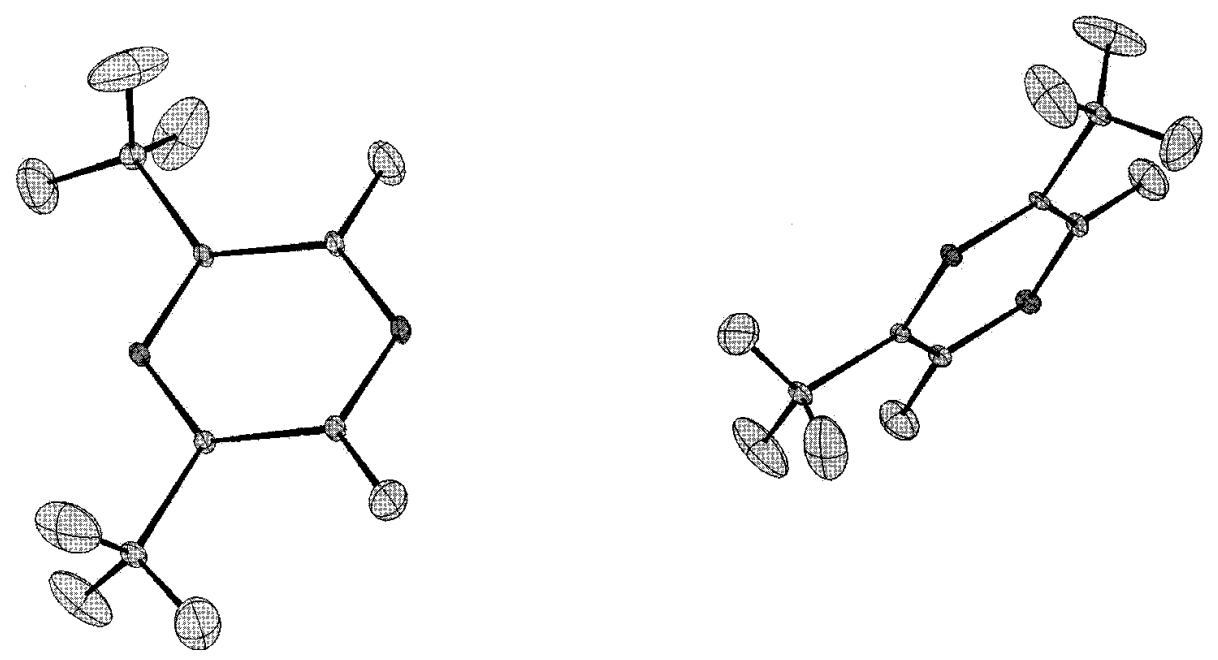

Arrangement des

molécules dans une

maille.

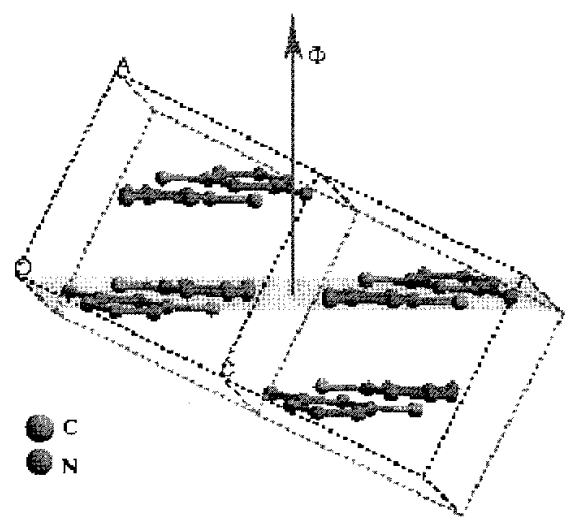

Les deux types de méthyle sont représentés ici par des couleurs différentes, les atomes d'hydrogène ne sont pas représentés. Le plan $\left(\begin{array}{lll}-2 & 0 & 1\end{array}\right)$ dans lequel ont été faites les mesures de diffusion inélastique des neutrons est représenté en jaune, l'axe [ $\left[\begin{array}{lll}-2 & 0 & 1\end{array}\right]$ autour duquel le monocristal a été tourné d'un angle $\phi$ est représenté en rouge. Cette orientation permet de 
mesurer le transfert de moment dans le plan (-2 0 1). Par mesure de plusieurs orientations sur IN10 (ILL) il a été possible d'attribuer sans ambiguité les fréquences observées à chacun des groupements méthyle.

La figure suivante, projection le long de l'axe a de la maille monoclinique, montre l'orientation relative des deux groupements méthyle (maille monoclinique, groupe d'espace $\mathrm{P} 21 / \mathrm{a}, \mathrm{a}=7.288(13) \AA, \mathrm{b}=10.725(15) \AA, \mathrm{c}=7.41(1) \AA$ et $\left.\beta=90.4(1)^{\circ}\right)$. On peut voir sur cette figure que les plans des protons liés aux carbones notés $\mathrm{C} 5$ sont pratiquement alignés tandis que les plans des protons liés aux carbones C6 forment entre eux des angles voisins de $60^{\circ}$ et $120^{\circ}$. Deux pics inélastiques à 20 et $29 \mu \mathrm{eV}$ ont été observés sur IN10B. La fréquence observée à $20 \mu \mathrm{eV}$ correspond aux protons du carbone noté $\mathrm{C} 5$ et celle à $29 \mu \mathrm{eV}$ à ceux du carbone C6.

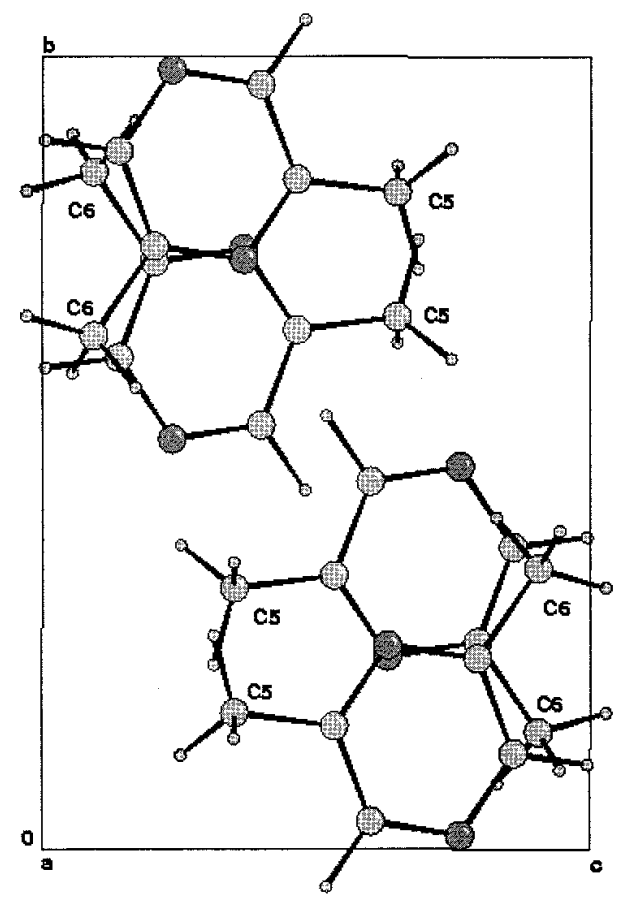

La connaissance de la structure à basse température et l'attribution des transitions observées permet ensuite d'interpréter les spectres de diffusion inélastique des neutrons, puis d'obtenir des informations sur la dynamique des rotateurs méthyles grâce aux simulations en comparant les potentiels calculés aux potentiels expérimentaux.

Dans ce cas, les résultats des calculs effectués par mécanique moléculaire et ab initio de type Hartree-Fock sont comparables aux données expérimentales quant à l'orientation du groupement méthyle dans le cristal, la forme et la hauteur de la barrière rotationnelle.

La méthode dela fonctionnelle de densité (DFT), très coûteuse en temps de calcul, fournit des résultats excellents. Il semble que généralement la DFT offre une estimation du potentiel beaucoup plus fiable pour une grande variété de molécules organiques pour lesquelles les méthodes $a b$ initio et de mécanique moléculaire se sont révélées insuffisantes. Cependant cette méthode semble fournir très peu d'information sur l'origine du potentiel 
rotationnel (van der Waals, Coulomb), ce qui rend difficile l'interprétation physique des résultats.

$$
\omega=20 \mu \mathrm{EV}
$$

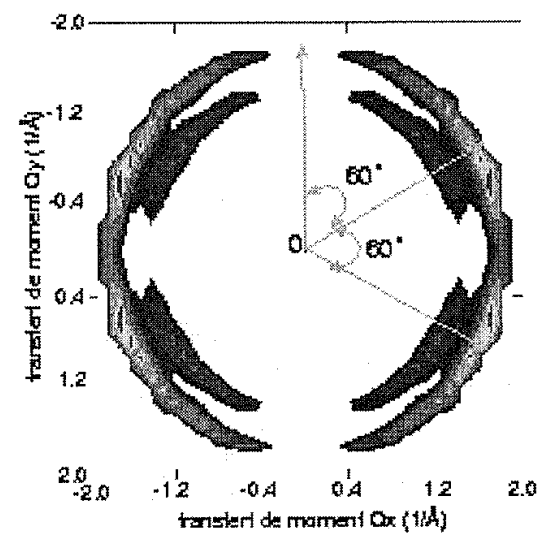

$\omega=29 \mu \mathrm{EV}$

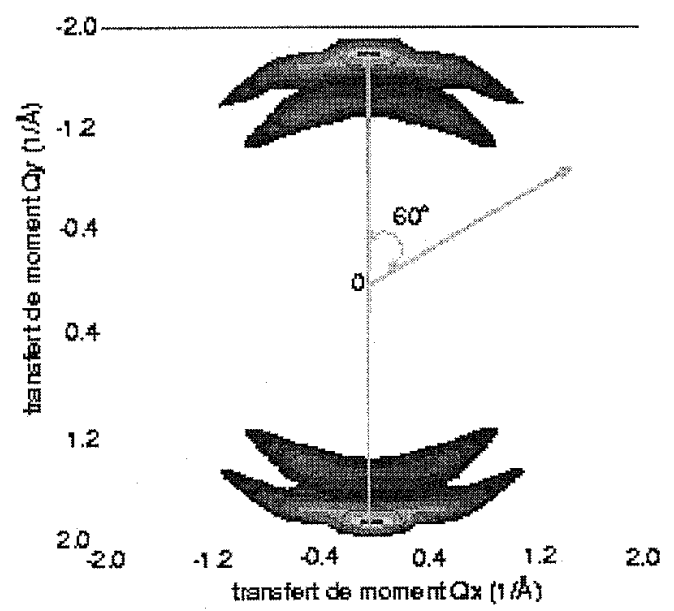

Cartes $S\left(Q_{x}, Q_{y}, \omega\right)$ reprisentant l'intensité du pic tunnel situé à ? en fonction du transfert de moment. Par souci de clarté, seule l'intensité autour des maxima est représentée.

Articles et thèses : B. Nicolaï et al., thèses B. Nicolaï et E. Kaiser Morris. 


\subsubsection{L'acétate de lithium $\mathrm{LiCH}_{3} \mathrm{COO}, 2 \mathrm{H}_{2} \mathrm{O}$}

Le spectre d'effet tunnel de l'acétate de lithium dihydraté présente plusieurs transitions d'effet tunnel et a donné lieu à plusieurs interprétations contradictoires: méthyle isolé, couplage entre deux méthyles, chaîne infinie de méthyles couplés, couplage entre les mouvements de rotation du méthyle et de translation du centre de masse...

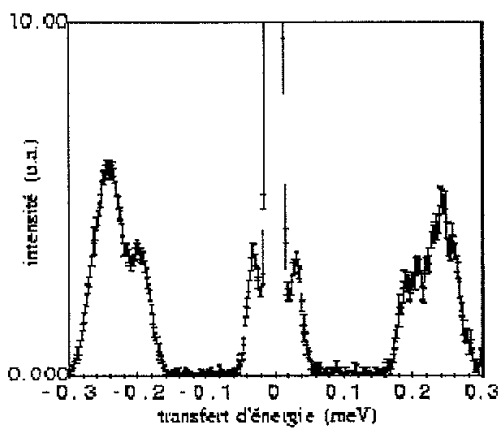

Spectre de diffusion inélastique des neutrons enregistré à 1,7K sur IN5 (ILL) avec une longueur d'onde incidente de 10,4A. L'échantillon est une poudre d'acétate de lithium dihydraté. L'intensité est en unités arbitraires. Un pic principal apparaît à $250 \mu \mathrm{eV}$, pic clairement divisé en au moins 2 pics disctincts. Un pic inélastique plus faible est visible à $40 \mu \mathrm{eV}$.

Une étude sur le composé totalement deutéré $\mathrm{LiCD}_{3} \mathrm{COO}, 2 \mathrm{D}_{2} \mathrm{O}$ révèle une transition de phase, non observée sur le composé totalement hydrogéné $\mathrm{LiCH}_{3} \mathrm{COO}, 2 \mathrm{H}_{2} \mathrm{O}$. Contrairement aux CH3, les CD3 deviennent ordonnés à basse température. Cependant, les effets de la deutération des groupements méthyles et de la deutération des molécules d'eau ne peuvent pas être distingués. Pour cela, nous avons étudié le composé partiellement deutéré $\mathrm{LiCD}_{3} \mathrm{COO}, 2 \mathrm{H}_{2} \mathrm{O}$.

Etude de la structure cristalline de $\mathrm{LiCD}_{3} \mathrm{COO}, 2 \mathrm{H}_{2} \mathrm{O}$ par diffraction de neutrons
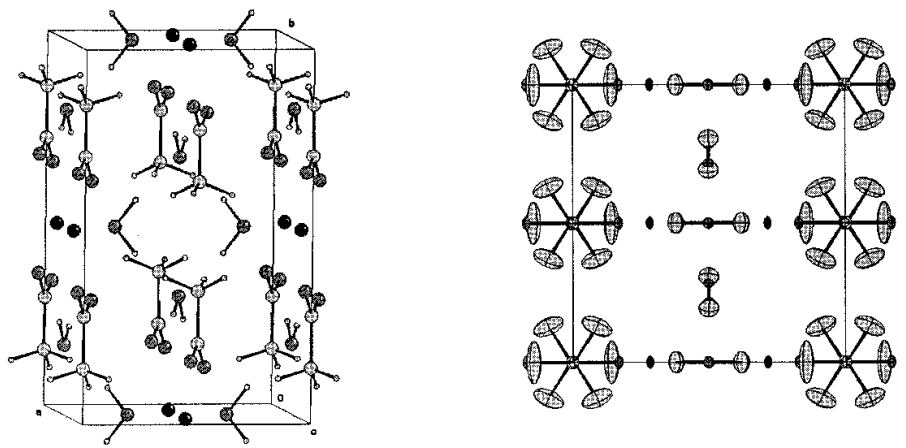

Représentation de la structure de l'acétate de lithium deutéré dihydraté à $14 \mathrm{~K}$. La figure de droite est une projection de la structure suivant l'axe b et montre la conformation étoilée des atomes de deutérium. 
L'étude sur monocristal, à 300,40 et $14 \mathrm{~K}$, révèle une transition de phase à $17.5 \pm$ $0.5 \mathrm{~K}$, analogue à celle du composé totalement deutéré: les $\mathrm{CD}_{3}$ deviennent ordonnés à basse température. Cela démontre clairement le rôle prédominant de la deutération des groupements méthyles dans la transition de phase. A notre connaissance cet effet n'a jamais été observé pour des systèmes contenant des groupements méthyles.

Les cartes de Fourier des groupements méthyles nous fournissent une représentation graphique des effets de la température sur la localisation des atomes de deutérium.

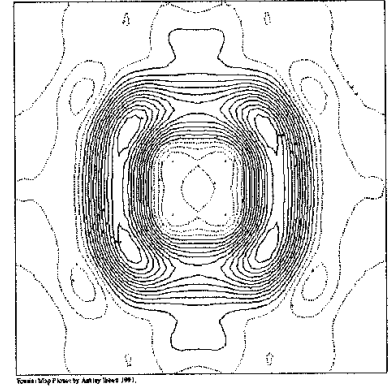

$300 \mathrm{~K}$

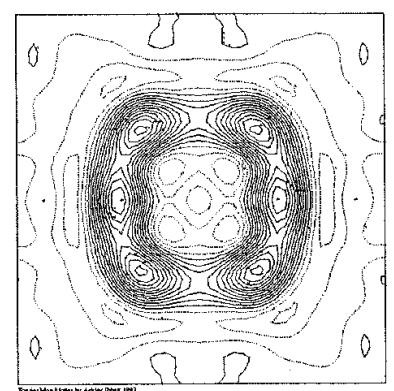

$40 \mathrm{~K}$

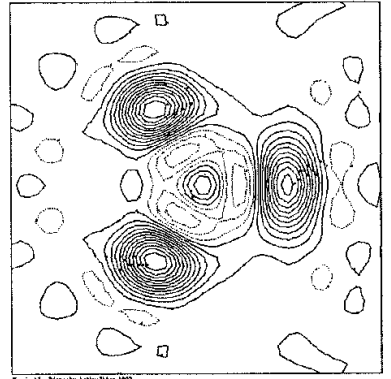

$14 \mathrm{~K}$

A $300 \mathrm{~K}$ et à $40 \mathrm{~K}$ il s'agit de séries-différences de Fourier dans le plan de l'anneau, et à $14 \mathrm{~K}$ d'une transformée de Fourier dans le plan des atomes de deutérium.

On remarque :

- qu'à 300K, la distribution des atomes de deutérium est relativement diffuse, on peut conclure à une libre rotation des groupements méthyles ;

- qu'à 40K, on distingue six maxima : cela peut correspondre soit à un méthyle dans un potentiel de symétrie six, soit à la superposition de méthyles dans des potentiels de symétrie d'ordre trois et ayant des orientations différentes et équiprobables ;

- enfin qu'à $14 \mathrm{~K}$, les $\mathrm{CD}_{3}$ sont localisés. Les groupements $\mathrm{CD}_{3}$ sont dans un potentiel de symétrie trois avec une barrière relativement élevée. Cependant il n'y a aucun réarrangement significatif des positions atomiques $(\mathrm{Li}, \mathrm{C}, \mathrm{O}, \mathrm{H})$ à la transition de phase : en conséquence les potentiels à $40 \mathrm{~K}$ et à $14 \mathrm{~K}$ devraient être quasiment identiques.

Jusqu'à présent, il n'y a aucun modèle qui rende compte de l'effet de la deutération sur la localisation des méthyles. Par conséquent les effets de masse et les comportements quantiques différents des $\mathrm{CH}_{3}$ et $\mathrm{CD}_{3}$ doivent être considérés. 


\subsubsection{Le diacétate de manganèse tetrahydraté}

La structure du composé $\mathrm{Mn}\left(\mathrm{C}_{2} \mathrm{H}_{3} \mathrm{O}_{2}\right)_{2} \cdot 4 \mathrm{D}_{2} \mathrm{O}$ a été déterminée à $300 \mathrm{~K}$ puis à $14 \mathrm{~K}$ dans le système monoclinique, groupe d'espace $\mathrm{P} 21 / \mathrm{c}$.

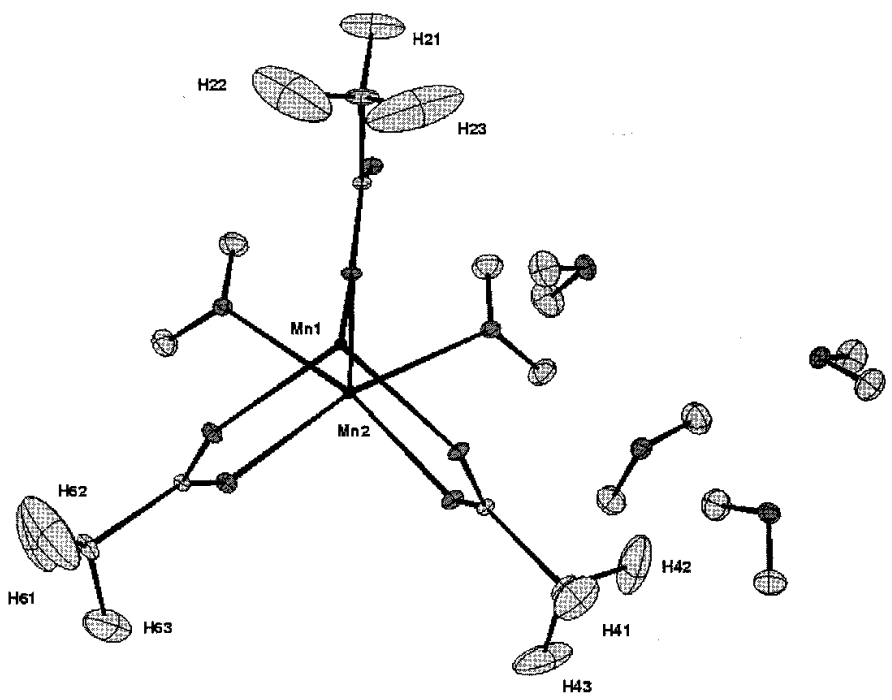

Représentation des atomes de l'unité asymétrique et des ellipsoïdes à $14 \mathrm{~K}$.

De la même manière que précédemment, des mesures de diffusion de neutrons (IN5 à l'ILL) sur deux monocristaux orientés ont permis d'attribuer à chaque $\mathrm{CH}_{3}$ les trois transitions d'effet tunnel observées à 1.2, 50 et $137 \mu \mathrm{eV}$.
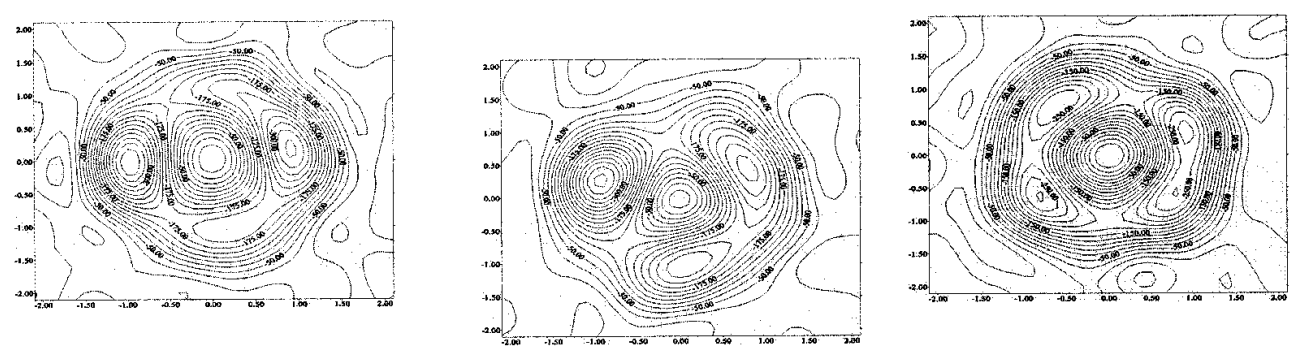

Distribution des protons à $300 \mathrm{~K}$, de gauche à droite liés à $C_{2}, C_{4}$ et $C_{6}$.

Cette figure montre le caractère diffus de la distribution des protons à température ambiante. Le modèle de l'anneau a été utilisé pour affiner ces distributions. Comparé à un affinement à partir d'une répartition de protons sur des positions discrètes, le nombre de 
paramètres à affiner est réduit (314 au lieu de 379) et le coefficient d'accord $w R$ est signicativement meilleur $(4,56 \%$ au lieu de $7 \%)$.

On remarque sur ces figures une irrégularité dans la distribution, un écart certain par rapport à un modèle de libre rotation. Pour le méthyle $C_{4}$ la symétrie trois suggère une localisation résiduelle des protons. La barrière de potentiel devrait être plus grande que l'énergie thermique. La distribution des méthyles $\mathrm{C}_{2}$ et $\mathrm{C}_{6}$ correspond davantage à une rotation quasi libre. On remarque toutefois deux faibles extrema de densité pour $\mathrm{C}_{2}$ et quatre pour $\mathrm{C}_{6}$ incompatibles dans les deux cas avec des rotateurs simples libres ou des potentiels de symétrie ternaire. Des distributions semblables ont déjà été observées et par exemple dans le cas de l'acétate de lithium interprétées en terme de couplage entre la rotation du groupe méthyle et la translation du centre de masse.

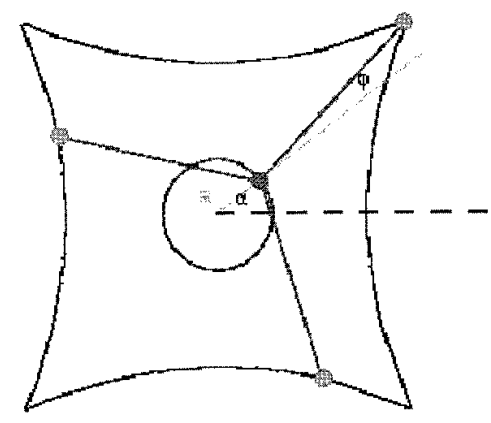

Le cercle rouge représente la trajectoire du centre de masse des trois protons représentés en bleu, en raison du couplage la trajectoire des protons décrit plus un carré qu'un cercle.

A basse température les protons sont plutôt bien localisés. Le modèle de l'anneau n'est plus adéquat et un affinement classique a été réalisé. Les cartes de densité des protons des méthyles $\mathrm{C} 4$ et $\mathrm{C} 6$ correspondent assez bien à des rotateurs gênés avec des barrières de potentiel élevées et une symétrie ternaire. La localisation des protons du C4 comparée à celle $\mathrm{du} \mathrm{C} 6$, suggère une barrière de potentiel plus grande. La densité de protons du groupement méthyle $\mathrm{C} 2$ est également compatible avec des protons localisés, mais présente un écart important à la symétrie ternaire, un proton est plus localisé que les deux autres. Ce type de distribution ne semble pas avoir été jamais signalé auparavant.

Des calculs classiques de dynamique moléculaire ont été effectués alors pour représenter la dynamique des groupes méthyles. La complexité de la structure et le volume relativement important de la maille élémentaire limitent les possibilités de simulations. L'utilisation du champ de force universel conduit à des résultats satisfaisants et en particulier reproduit bien la distribution observée pour le méthyle $\mathrm{C} 2$ avec un écart significatif par rapport à la symétrie ternaire. La simulation montre que cette distribution particulière pourrait être reliée à l'anisotropie des déplacements de l'atome $\mathrm{C} 2$ : 1'amplitude calculée perpendiculairement au plan $b c$ est plutôt importante. Cependant, la distribution est différente de celle correspondant à un couplage entre rotation et translation.

Une approche purement quantique a également été développée et rend compte des distributions observées.

Voir en référence : article B. Nicolaï et al., P. Schiebel et al., thèse B. Nicolaï. 
Di-acétate de manganèse tétrahydraté : cartes de densités dans le plan des protons des méthyles C4, C6 et C2 : $j^{\text {ere }}$ ligne carte $3 D$ inversée, $2^{\text {nde }}$ ligne carte $2 D, 3^{\text {ème }}$ ligne carte calculée à partir de la mécanique quantique et $4^{\text {ème }}$ ligne carte calculée par la dynamique moléculaire.
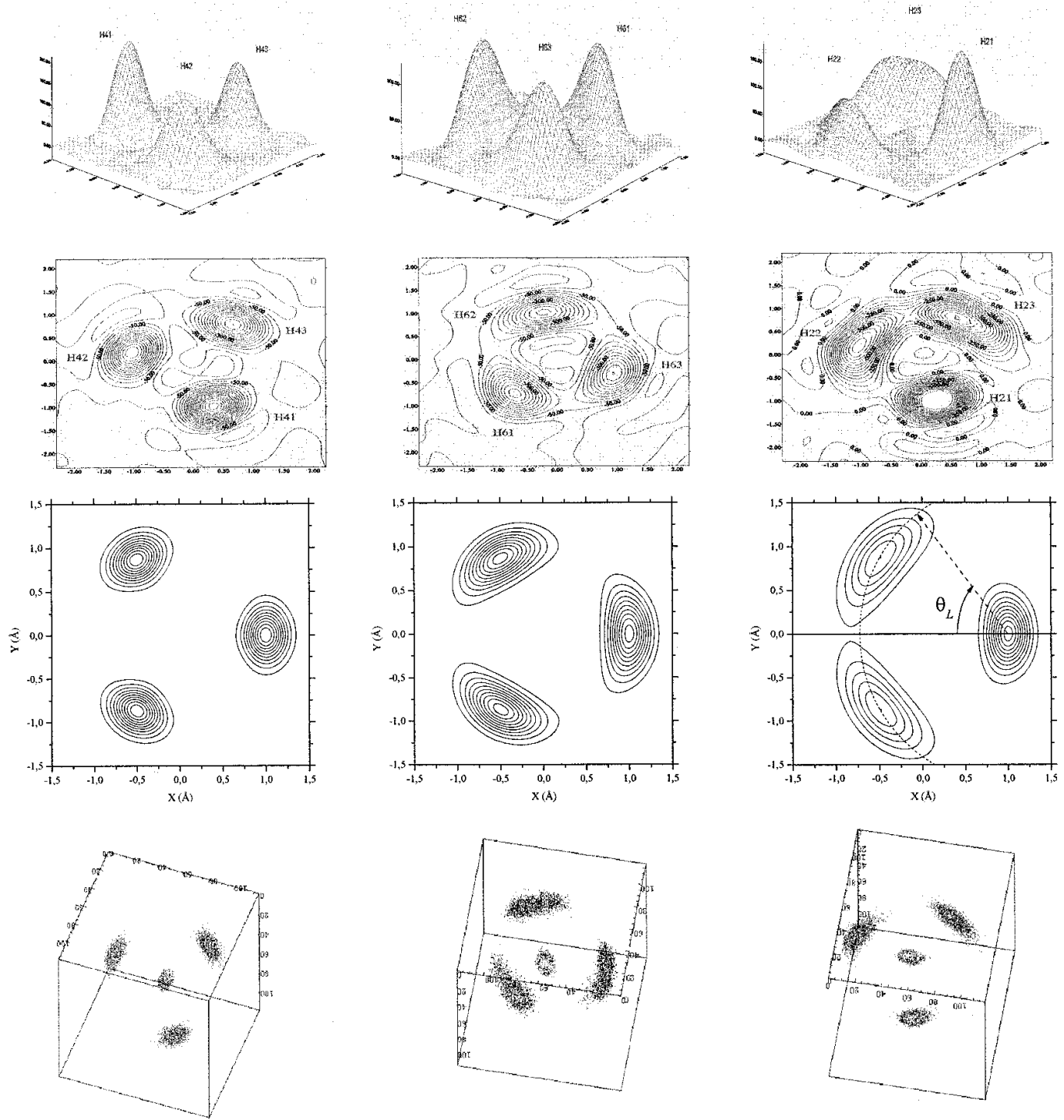

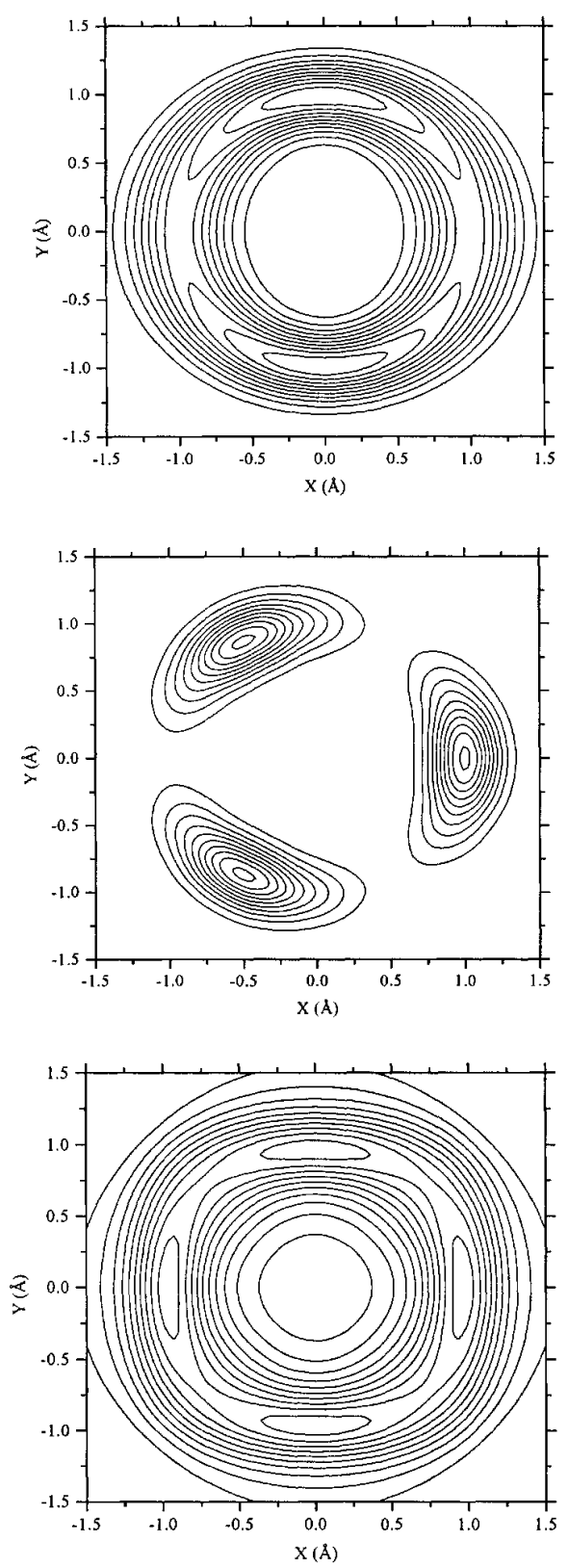

Diacétate de manganèse tétrahydraté à $300 \mathrm{~K}$ : cartes de densité des protons calculées à partir du potentiel vu par chaque méthyle convolué par la distribution statistique des atomes de carbone auxquels ils sont liés, de haut en bas pour les groupes méthyle C2, C4 et C6. 


\subsubsection{La $\gamma$-picoline ou 4-méthyl pyridine}

Certainement une des petites molécules les plus étudiées, la $\gamma$-picoline possède quelques propriétés qui la rendent attachante : hygroscopique avec un point de fusion aux alentours de $-6^{\circ} \mathrm{C}$, elle cristallise dans le groupe d'espace I41/amd pour très vite se macler en I41/a à la transition du second ordre, aux environs de $254 \mathrm{~K}$ et après quelque temps passé en chambre froide en sa compagnie elle devient franchement inoubliable.

Quelle que soit la température $(260 \mathrm{~K}, 220 \mathrm{~K}, 120 \mathrm{~K}, 20 \mathrm{~K}$ et $10 \mathrm{~K})$ le seul groupement méthyle apparaît comme un rotateur quasi libre.

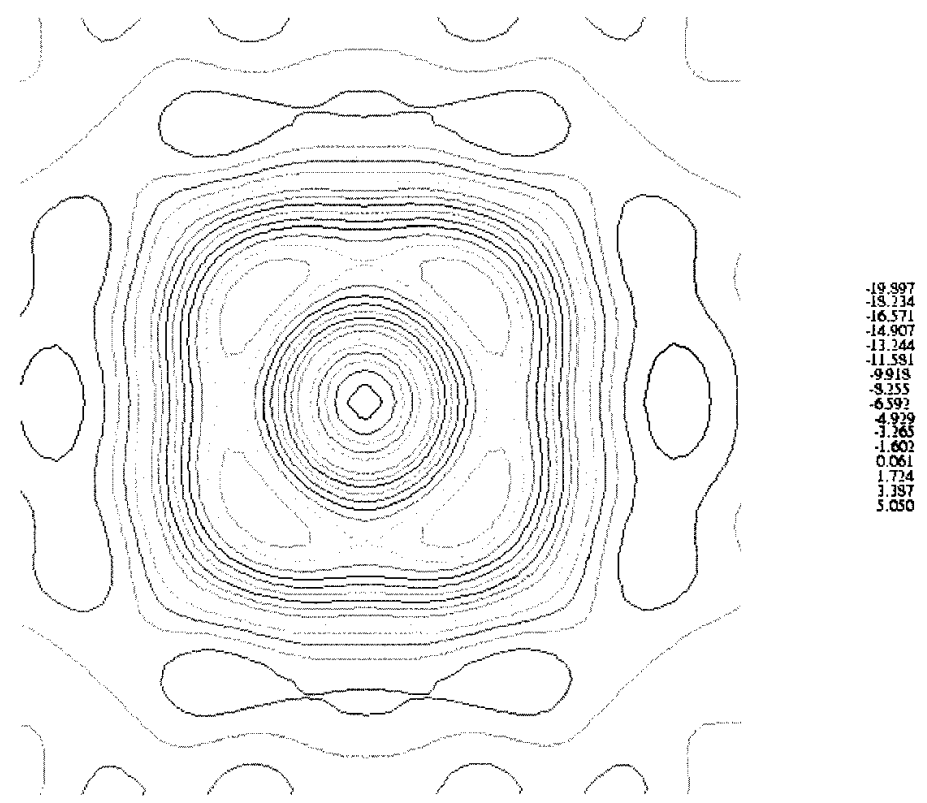

Carte de densité à $260 \mathrm{~K}$ : anneau avec des minima aux sommets d'un carré. La répartition de la densité est indiquée sur l'échelle.

C'est le premier système moléculaire pour lequel furent observées, dès 1975 , les excitations quantiques du groupe méthyle. Depuis ce composé n'a pas cessé d'être étudié, la seule étude structurale par diffraction de neutrons sur monocristal à $120 \mathrm{~K}$ rapporte que le groupe méthyle est en désordre dynamique et indique que la rotation de ce dernier se fait dans un potentiel de symétrie d'ordre 6 .

L'étude structurale en-dessous de la transition doit tenir compte du maclage, passage du groupe de Laue $4 / \mathrm{mmm}$ à $4 / \mathrm{m}$ et du groupe d'espace I41/amd à I41/a. L'affinement se fait impérativement sur les $\mathrm{F}^{2}$, les facteurs de structure n'étant pas additifs. Connaissant la loi de macle, il est possible, après affinement, d'éliminer par le calcul la somme des contributions des domaines correspondant à un individu de la macle. Bien que les erreurs deviennent plus importantes, car reportées sur l'individu restant, il est alors possible de calculer des cartes de Fourier, spécialement dans le plan des protons. 


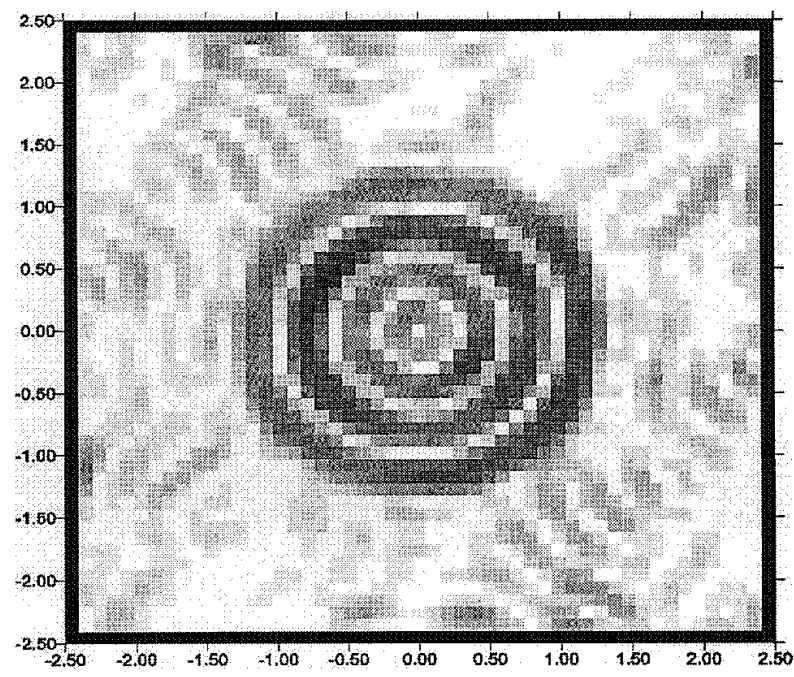

Carte de densité dans le plan des protons de la భpicoline à $10 \mathrm{~K}$.

A $10 \mathrm{~K}$ on remarque immédiatement que ça tourne rond, contrairement à $260 \mathrm{~K}$. L'analyse des paramètres thermiques en corps rigide, sans les protons, montre que les modules de la libration varient régulièrement entre 10 et $260 \mathrm{~K}$. La libration principale a lieu autour de l'axe $c$, l'amplitude passe de $2,4^{\circ}$ à $10 \mathrm{~K}$ à $14,3^{\circ}$ à $260 \mathrm{~K}$. Les deux autres mouvement de libration sont beaucoup plus faibles. Le plan moyen des molécules est dans les plans miroirs principaux à haute température. A la transition de phase les molécules tournent autour de l'axe $c$. On observe une diminution notable de la libration dans cette direction, environ $10 \%$ et la perte des miroirs diagonaux. L'angle entre le plan moyen de la molécule et par exemple le plan $\left(\begin{array}{lll}1 & 0 & 0\end{array}\right)$ varie brutalement à la transition puis de manière continue et se stabilise $13.5(1)^{\circ}$ à $10 \mathrm{~K}$.

La distance $\mathrm{CH}_{3}-\mathrm{CH}_{3}$ la plus courte apparait le long de l'axe c. Cette distance, plus courte que la somme des rayons de van der Waals, $4 \AA$, justifie a priori l'application de la théorie du couplage entre deux rotateurs au cas de la $\gamma$-picoline. Perpendiculairement à l'axe c, la distance $\mathrm{CH}_{3}-\mathrm{CH}_{3}$ est de $3,9 \AA$. Les méthyles forment deux chaînes infinies de méthyles équidistants, parallèles à l'axe a et à l'axe $b$.

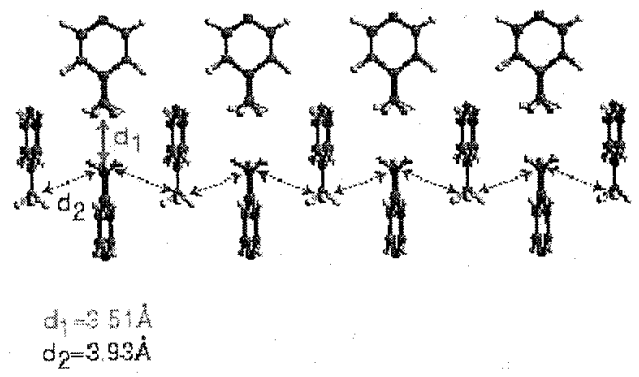

Représentation des chaînes de groupements méthyles dans la భpicoline. 
Parce que les modèles de couplage avec un autre rotateur ou avec les phonons ne décrivent pas de façon satisfaisante les spectres de diffusion neutronique des mélanges isotopiques $\left(\mathrm{CH}_{3}-\mathrm{CD}_{3}\right)$ ou de molécules partiellement deutérées de la $\gamma$-picoline, un troisième modèle de couplage a ensuite été développé : le modèle de chaînes infinies de rotateurs couplés, en bon accord avec des études Raman à basse température qui suggérèrent les premières une dynamique collective des groupements méthyles. Cette interpétation utilise le formalisme quantique de sine-Gordon. Ce potentiel de sine-Gordon a également été appliqué avec succès dans le cas de deutération partielle de la molécule, puis aux cas de l'acétate de lithium dihydraté et de la 2,6 diméthylpyridine (ou lutidine).
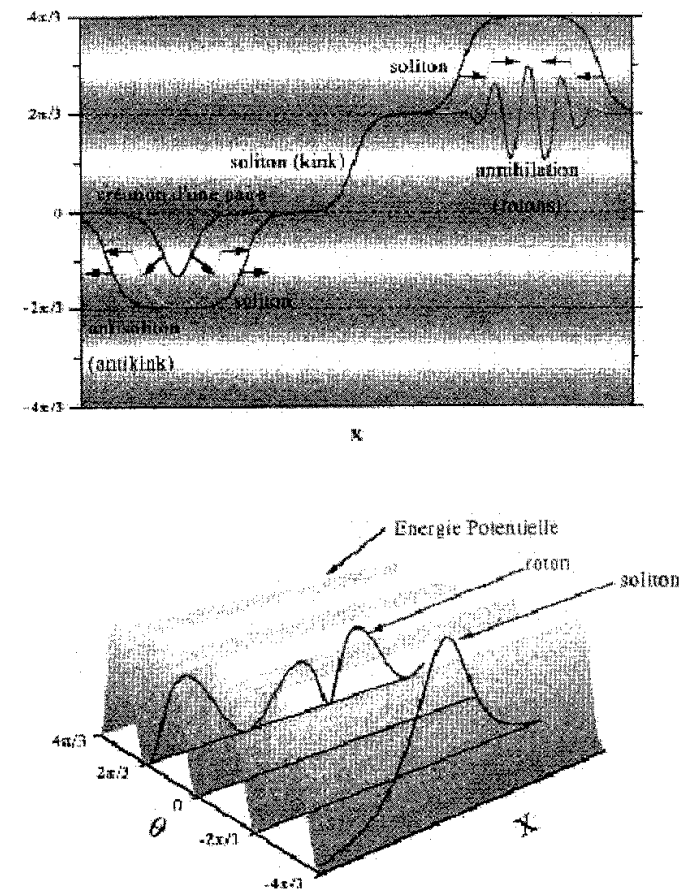

Représentation des excitations dans la chaîne en fonction de $\theta$ l'orientation du méthyle et X la position dans la chaîne

Le soliton fait passer la moyenne des groupements méthyle d'un minimum du potentiel local vers un autre minimum. Le breather est une paire liée soliton-antisoliton.

Des calculs récents de simulation numérique ont également été réalisés mettant en jeu un couplage rotation-précession et un couplage entre rotateurs. Dans ce cas les potentiels de couplage et la trajectoire de rotation-précession ont été déterminés par des calculs de mécanique moléculaire.

Voir en référence les articles de François Fillaux, les thèses de B. Nicolaï et M. Neumann. 


\section{CONCLUSION}

Les quelques exemples cités montrent l'apport de la diffraction de neutrons à l'étude de la dynamique des $\mathrm{CH}_{3}$. Bien que ces études soient très complètes, les perspectives sont nombreuses.

Le trichloromésitylène présente une transition de phase, peut-être reconstructive.

Il est nécessaire de développer un modèle théorique si on veut interpréter la transition de phase dans l'acétate de lithium deutéré dihydraté.

La répartition des charges, la charge effective des atomes dans les composés moléculaires et les complexes porteurs de groupements méthyles constituent un des paramètres les moins bien connus. Une réponse ici pourrait être d'utiliser également les rayons $\mathrm{X}$ (méthode $\mathrm{X}-\mathrm{N}$ ).

La deutération partielle du groupe méthyle $\left(\gamma\right.$-picoline $\left.\mathrm{CH}_{2} \mathrm{D}\right)$ permet de distinguer entre dynamique isolée et collective. Une transition de phase à $5,4 \mathrm{~K}$ a été mise en évidence pour ce composé, un monocristal a été étudié à $100 \mathrm{~K}$ au LLB, passera-t-il l'été avant la suite à l'ILL ? ?

La $\mathrm{N}$-oxypicoline présente un spectre complexe à basse température, au moins quatre transitions tunnel. A $300 \mathrm{~K}$ la structure est maintenant établie, un seul $\mathrm{CH}_{3}$ indépendant en rotation libre. La description à $10 \mathrm{~K}$ attendra que la transition du second ordre entre 120 et $100 \mathrm{~K}$ puis la transition du premier ordre à $90 \mathrm{~K}$ soient parfaitement comprises. La lutidine également présente deux transitions de phase, vues en diffraction sur poudres. C'est peu par rapport aux cinq transitions annoncées par les spectroscopistes.

Finalement n'oublions pas que le proton se rencontre dans d'autres systèmes, la liaison hydrogène c'est lui, et que lorsqu'il n'occupe pas plusieurs sites cristallographiques, il n'est pas pour autant toujours où on l'imagine.

\section{REFERENCES}

\section{Sites web}

http://journals.iucr.org (sans obligation de publier une structure dans Acta Cryst. le test (checkcif) est très utile et gratuit).

http://www.xtl.ox.ac.uk (pour installer CRYSTALS à partir de CRYSTALS SOFTWARE sur ce site).

\section{Ouvrages}

- International Tables for X-ray Crystallography (tous volumes, toutes éditions).

- Neutron Diffraction. G.E. Bacon, Oxford University Press, ISBN 198513534

- Single Crystal Neutron Diffraction From Molecular Materials. Chick C Wilson. Published by World Scientific Publishing Co. Pte. Ltd. ISBN 981-02-3776-6 (8 chapitres et 935 références).

- Théorie et Technique de la Radiocristallographie. A. Guinier. Dunod Paris 1964.

- Crystal-Structure Analysis. M.J. Buerger. Wiley, New York, 1960.

- Direct Methods in Crystallography. C. Giacovazzo. Academic Press Inc. (London) LTD. ISBN 0-12-282450-4

- Fundamentals of Crystallography. International Union of Crystallography, Oxford University Press. C. giacovazzo, H.L. Monaco, D. Viterbo, F. Scordari, G. Gilli, G. Zanotti, M. Catti. ISBN 0198555792 (Hbk) ISBN 0198555784 (Pbk)

- Mathematical Techniques in Crystallography and Materials Science. E. Prince. ISBN 3-54058115-4 Springer-Verlag Berlin Heidelberg New York.

- Springer Tracts Modern Physics. W. Press (1981) Vol. 92. New York : Springer Verlag. 
- Rotational Tunnelling Spectroscopy with Neutrons. C.J. Carlile and M. Prager. " Condensed matter and materials science with neutron beam techniques " Editors: U. Dahlborg and S.W. Lovesey. International Journal of Modern Physics B.

- Cours de Cristallographie. (4 volumes) R. Gay, Paris, Gauthier-Villars, 55 Quai des GrandsAugustins, 1959.

- Transformées de Fourier, manuel pratique. Tournarie et Curien.

\section{Proceedings}

- Proceedings of the $X$ Workshop on Quantum Atomic and Molecular Tunnelling in Solids. Kraków, 26-30 septembre 1999. ISBN 83-87671-78-9. ISSN 1505-1250.

- The access to molecular disorder by diffraction : a tutorial. W. Prandl and P. Schiebel, Molecular Physics Reports 31 (2001) 9-33.

- Inelastic neutron scattering study of the quantum Sine-Gordon breather in 4-methylpyridine. F. Fillaux. Molecular Physics Reports 31 (2001) 60-71.

\section{Revue}

- L'effet tunnel et les protéines par Mark Johnson et Gordon Kearley. Pour la Science, édition française de Scientific American, mars 2001, 64-69.

\section{Thèses}

- Dynamique et désordre du groupe méthyle dans les différentes phases de la 2,6-diméthyle pyrazine, 4-méthyle pyridine et 4-méthyle pyridine $N$-oxyde. Erika Kaiser Morris, Université Paris XI, 1997.

- Dynamique rotationnelle de groupements méthyles et structure cristalline à basse température: étude par diffusion des neutrons et modélisation. Béatrice Nicolaï, Université Paris 7 - Denis Diderot, 1998.

- Etude des trihalogénomésitylènes: structure, vibrations moléculaires, dynamique des groupes méthyles. Fahima Boudjada, Université de Rennes 1, 1999.

- Le groupement méthyle - sonde de l'énergie potentielle. Marcus A. Neumann, Université Joseph Fourier-Grenoble 1, 1999.

- Structure et dynamique quantique de cristaux moléculaires: spectroscopie et modélisation numérique. Marie Plazanet, Université Joseph Fourier-Grenoble 1, 2000.

\section{Quelques articles}

- Alefeld B., Kollmar A., Dasannacharya B.A. J. Chem. Phys. (1975) 63, No. 10, 4415-4417.

- Carlile C.J., Ibberson R.M., Fillaux F. and Willis B.T.M. Zeitschrift für Kristallographie 193 (1990) 243-250.

- Chernyshev V.V., Zhukov S.G., Yatsenko A.V., Aslanov L.A. Acta Cryst. (1994). A50, 601605.

- Fillaux F. and Carlile C.J. Chemical Physics Letters Vol. 162, No 3 (1989) 188-195.

- Fillaux F. and Carlile C.J. Physical Review B Volume 42, Number 10, (1990) 5990-6006.

- Fillaux F., Carlile C.J., Kearley G.J. Physical Review B, 44, No 22 (1991) 12280-12293.

- Fillaux F., Carlile C.J., Kearley G.J., Prager M. Physica B 202 (1994) 302-310.

- Kearley G.J. et Nicolaï B. Méthodes numériques et simulations. J. Phys. IV France 10 (2000) 237-249.

- Kearley G.J., Nicolaï B., Radaelli P.G. and Fillaux F. Journal of Solid State Chemistry 126 (1996) 184-188. 
- Kiat J.M., Baldinozzi G., Dunlop M., Malibert C., Dkhil B., Ménoret C., Masson O. and Fernandez-Diaz M.T. J. Phys. : Condens. Matter 12 (2000) 8411-8425.

- Kuhs W.F. (1992). Acta Cryst. A48, 80-98.

- Meinnel J., Häusler W., Mani M., Tazi M., Nusimovici M., Sanquer M., Wyncke B., Heidemann A., Carlile C.J., Tomkinson J. and Hennion B. Physica B 180 \& 181 (1992) 711713.

- Meinnel J., Mani M., Tonnard F., Nusimovici M. et Sanquer M. C. R. Acad. Sci. Paris, t. 317, Série II (1993) 885-890.

- Meinnel J., Mani M., Nusimovici M., Carlile C.J., Hennion B., Carrié R., Wyncke B., Sanuer M., Tonnard F. Physica B 202 (1994) 293-301.

- Meinnel J., Hennion B., Mani M., Wyncke B., Carlile C.J. Physica B 213 \& 214 (1995) 649651.

- Meinnel J., Carlile C.J., Knight K.S., Godard J. Physica B 226 (1996) 238-240.

- Meinnel J., Mani M., Cousson A., Boudjada F., Paulus W., Johnson M. Chemical Physics 261 (2000) 165-187.

- Müller-Warmuth, Schüler R., Prager M. and Kollmar A. J. Chem. Phys. Vol. 69, No 6, (1978) 2382-2392.

- Nicolä̈ B., Kaiser E., Fillaux F., Kearley G.J., Cousson A., Paulus W. Chemical Physics 226 (1998) 1-13.

- Nicolaï B., Kearley G.J., Cousson A., Paulus W., Fillaux F., Gentner F., Schröder L. and Watkin D. Acta Cryst. (2001). B57, 36-44.

- Noël H., Le Marouille J-Y. Journal of Solid State Chemistry (1984). V. 52(3) 197-202.

- Ohms U., Guth H., Treutmann W., Dannöhl H., Schweig A., Heger G. J. Chem. Phys. Vol. 83, No. 1 (1985) 273-279.

- Schiebel P., Prand1 W., Papoular R. and Paulus W. Acta Cryst. (1996) A52, 189-197.

- Schoemaker V., Trueblood K.N. (1968) Acta Cryst. B24, 63.

- Sears V.F. (1992) Neutron News, Vol. 3, No. 3, 26-37.

- Tazi M., Meinnel J., Sanquer M., Nusimovici M., Tonnard F. and Carrié R. Acta Cryst. (1995) B51, 838-847.

\section{Programmes}

- CRYSTALS : Watkin D.J., Prout C.K., Carruthers J.R. \& Betteridge P.W., 1996, Chemical Crystallography Laboratory, Oxford, England. (une nouvelle version est actuellement disponible sur le site web cité précédemment, programme d'affinement gratuit accompagné de programmes de résolution de structures et d'un logiciel graphique en ligne CAMERON Watkin D.J., Prout C.K. \& Pearce L.J., 1996, Chemical Crystallography Laboratory, Oxford, England).

- Molecular Simulations Inc. (1996). Cerius2. BIOSYM/Molecular Simulations Inc. San Diego, CA 92121-3752, USA.

\section{Remerciements}

Gilles André, Burkhard Annighöfer, Jeanine Borjet, François Fillaux, Jean Godard, Jean Meinnel, Béatrice Nicolaï, Henri Noël, Werner Paulus, Christian Scherf, Ludger Schröder, David Watkin et bien sur CNRS et CEA. 